\title{
Enhanced S-MAC Protocol for Early Reaction and Detection in Wireless Video Sensor Networks
}

\author{
Christian Salim $^{a, b}$, Amani Srour $^{b}$, Rony Darazi $^{b}$, Abdallah Makhoul $^{a}$ and Raphaël Couturier ${ }^{a}$ \\ ${ }^{a}$ FEMTO-ST Institute, Univ. Bourgogne Franche-Comté, CNRS, France, Email: firstname.lastname@univ-fcomte.fr \\ ${ }^{b}$ TICKET Lab, Antonine University, Lebanon, Email: firstname.lastname@ua.edu.lb
}

\begin{abstract}
Wireless sensor networks (WSNs) continue their ascending developement to be among the leaders of technology. Furthermore, images are of paramount importance in several applications based on WSNs. Capturing, processing and transmitting the image face several challenges, mainly because of their highly needed power consumption. The huge number of images sensed and transmitted in a Wireless Video Sensor Network (WVSN) increases the dataflow on the overall network. A WVSN consists of three different layers: the video-sensor node, the coordinator and the sink. Sending images at the same time from different sensor nodes to a coordinator causes several problems. Besides energy consumption and bandwidth usage that represent the two major challenges in WSN, the queue of images on the coordinator can cause slower detection of intrusions and thus slower reaction from the coordinator. These reasons increase the need of a mac-layer protocol to control the network. We propose a new modified communication protocol based on the S-MAC protocol. This solution consists of adding a priority bit to the S-MAC protocol. Our approach is validated by experimentation using raspberry pi 3 and by simulation in OMNET++.
\end{abstract}

Index Terms-Wireless Video Sensor Networks; Early Detection; Delay; Quick Reaction; MAC Layer protocol.

\section{INTRODUCTION}

Since their inception, Wireless Sensor Networks (WSN) became increasingly popular in scientific and industrial communities. Thanks to numerous advantages, this technology has been able to establish itself as a key player in current network architectures. Industrial productions, military applications, habitat monitoring, and multimedia transmission are just a few examples of a wide and varied range of possible applications for continuous monitoring offered by the WSN. However, the size of the sensors requires the use of a battery as a source of energy which implies a limited lifetime. The sensors are designed for the collection of information. As a result, the organization of the network has become a major problem in the WVSN (wireless video sensor networks) field due to the huge number of sensor nodes sending their frames to the coordinator in a specific area of interest. Several communication protocols have been proposed in order to organize this network, taking into account the energy consumption to extend the life of the network. Numerous MAC protocols for wireless sensor networks adopt "dutycycle" operation to save energy (S-MAC, T-MAC, B-MAC, X-MAC, WiseMAC, RIMAC, ContikiMAC, ...) [1],[2] while organizing the network. In fact, our work focuses on the transmission of images in a multimedia WSN under the constraint of limited energy. In any proposed scenario, the surveillance remains the most important criteria in our study, such as how to detect an intrusion on the sensor and coordinator levels and how to react accordingly. A bibliographic study of WSN, MAC layer protocols is given. A newer version of S-MAC protocol is developed in this paper. The main objective of our work is to look for the possibility to improve the performance of the sensor network for a better surveillance system. Fig 1 shows the architecture of WVSN.

Our paper is divided into 3 sections, the first introduces the state of the art in this specific domain, the second explains in detail the proposed improvements and the third represents the different results obtained.

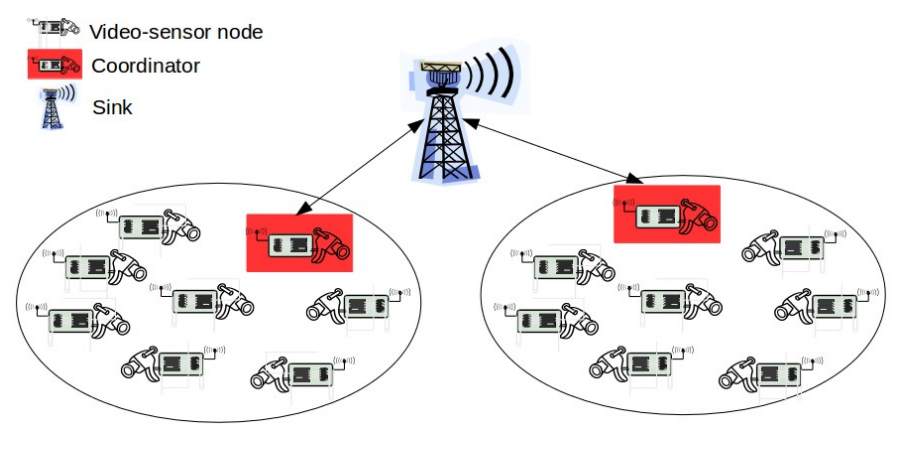

Fig. 1. Architecture of WVSN

\section{RELATED WORK}

In this section, several works from the literature working on the MAC-Layer protocol domain in WSN have been exploited.

Different categories of MAC Protocols can be found in the literature: Asynchronous, Synchronous and Hybrid schemes[3],[4],[5].

If the asynchronous schemes are adopted, every sensor becomes autonomous regarding its state (idle or active), so a wake up call is integrated in every sensor node to switch between active and sleeping states. It is defined by a specific MAC-Protocol applied in the network such as B-MAC [6], X-MAC [7], RI-MAC [8] and PW-MAC [9].

The synchronous category is divided into two sections, the locally synchronized and the globally synchronized [3]. S- 
MAC [10] and T-MAC [11] are two locally synchronized MAC-protocols that also adopt the duty cycling. Nodes are allowed to turn off their radio transmission module when no communication occurs during a certain time period to save energy related to transmission [1]. In the synchronous MACProtocols, all the neighboring nodes in a cluster wake up at the same time, which reflects the main difference with the asynchronous ones.

I-MAC [12] and Tree-MAC [13] are globally synchronized frame slotted MACs. They use the time-slot technique, dividing time into time slots and assigning time slots to nodes. The main problem of synchronized MACs is the high control overhead due to the synchronization of the network.

Several MAC-Protocols were designed to be QoS-aware (Quality of Service) for WSNs like in real time applications such as Q-MAC [14], PQ-MAC [15], RL-MAC [16] and CoSens [17]. To ensure a certain level of QoS by the application, additional challenges are imposed. Thus, flexibility and dynamicity are needed to react if any changes occur in the network [1] [18].

None of the above protocols takes into account the case when a critical frame is detected on a sensor node as mentioned in [19]. This frame must have priority over all the other frames in the coordinator's queue for quicker detection and reaction from the coordinator. In our approach, we are interested in adding a bit for criticality in the S-MAC protocol to be able to detect directly if the frame/image is critical or not. If the frame is critical, the protocol considers it has priority in the process over a non critical frame on the network. For data reduction in our approach, the sensing and transmission techniques are adopted from [19]. Sensor nodes are usually scattered in a capture area. Each of these nodes has the ability to collect data and route them to one or more coordinator nodes. The latter is a collection point for captured data. It can communicate the collected data to the end user through a communication network, possibly the Internet. Users can in turn use the base station as a gateway, in order to transmit their requests to the network. The network architecture is illustrated in Fig 1. The coordinator plays a key part when an intrusion is detected by one of its sensors. Its role is to react quickly so as no to miss any information about this intrusion (increasing the frame rate of the sensors, alerting the monitoring team ...).

\section{MASRA ALGORITHM}

In this section, we present a brief overview about the MASRA (Multimedia Adaptive Sampling Rate Algorithm) algorithm [19] where the network and sensor-nodes operate periodically. In our approach, the MASRA algorithm is used for data reduction on the sensor node level while sensing and transmitting data to the coordinator. In this algorithm, the frame rate of every video sensor-node varies in accordance with the number of critical frames sent all over a period. This frame rate increases when the number of critical frames increases and is set to a minimum of $1 \mathrm{FR} / \mathrm{s}$ in the passive state when there is no intrusion in the FOV (Field of View) of the sensor. Each sensor only sends the different frames to the coordinator depending on a color-edge similarity approach and a threshold of similarity. This approach helps reducing the data sensed and transmitted by the sensor nodes and thus reduces the energy consumption by more than $80 \%$ as in [19]. In the passive time, when the frame rate is equal to $1 \mathrm{FR} / \mathrm{s}$, each sensor sends the first frame of each period. A problem can occur in the passive sequence, if a zone of interest includes 200 sensor-nodes, and the $200^{\text {th }}$ sensor node detects an intrusion, this frame must be sent to the coordinator. It must wait in the queue of the coordinator so the 199 other first frames of the 199 other sensors get processed by the coordinator with a minimum of $50 \mathrm{~ms}$ for every frame. This problem leaves a gap of 5 seconds before a reaction from the coordinator. 10 seconds of non reaction can help an intrusion whose speed is $20 \mathrm{~m} / \mathrm{s}$ to riffle around 200 meters in the area of interest. To solve this problem, the critical frames must have a certain priority bit/flag to be processed before all the non critical frames.

\section{TheORETICAL APPROACH}

The S-MAC (Sensor MAC) protocol is based on the CSMA / CA (Carrier sense multiple access) method. It uses the RTS / CTS (Request to Send / Clear to Send) mechanism to deal with the problem of hidden nodes. S-MAC introduces a period of activity and standby as shown in Fig 2. Nodes must be synchronized in order to communicate. To do this, the nodes are organized in virtual clusters. Each node periodically broadcasts its scheduling program (its sleep and activity period) in a SYNC packet to other nodes in the same cluster. If a node belongs to two clusters, it must make a compromise between the two scheduling programs. S-MAC uses the concept of message passing, which allows fragments of a long message to be sent in a burst. As in the case of IEEE 802.11, the duration of the transmission is known by the NAV (Network Allocation Vector) which is included in the RTS and CTS control packets. S-MAC adds the duration of the remaining transmission in each fragment and in each ACK, this will allow the nodes that wake up in the middle of the transmission to return to the sleep state.

Several advantages emerge by adopting the S-MAC protocol:

- The loss of energy caused by listening to the free channel is reduced by the standby cycle.

- The protocol is simple.

\section{A. Ordering technique}

The communication between two nodes is characterized by the sending of signals: RTS and CTS indicating that a node has data to send (RTS: Ready to send / CTS: Clear to Send) and that a node is ready to receive.

1. Each node chooses its active and inactive periods according to its neighbors.

2. The first node to start is the only one freely choosing its periods, and then periodically announces its periods by sending synchronization signals (SYNC) during a dedicated period (at 


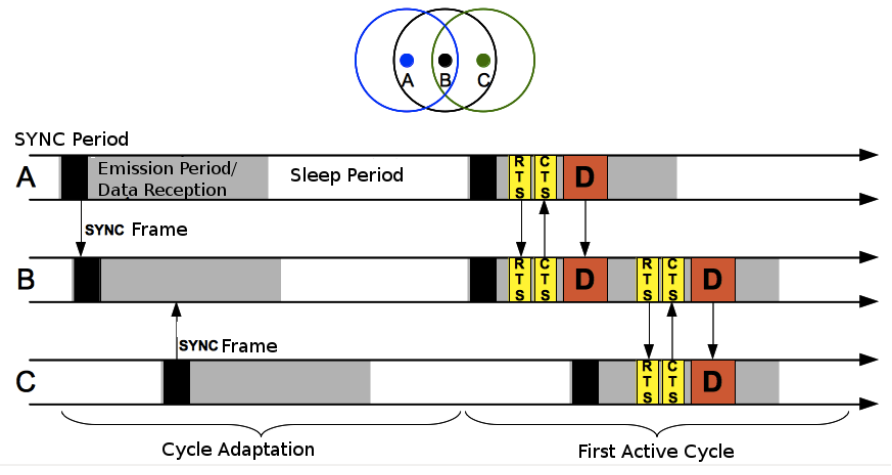

Fig. 2. S-MAC Period of activity and standby

the beginning of the active period).

3. The other nodes will adapt their own periods to those announced by the previous nodes. This adaptation mechanism is called "adaptive listening".

4. In Fig 2 Node C, the last one to start the "adaptive listening", adapts its active period to that of its neighbour B.

5. In Fig 2, we assume node $C$ is the last one to send its first frame of the period to the coordinator in the idle mode (Frame Rate $=1 \mathrm{FR} / \mathrm{s})$. Each node (A and B) has sent a frame to the coordinator before $\mathrm{C}$. The frame sent by $\mathrm{C}$ must wait for the coordinator to process the other 2 frames the wait time (delay) is given as follow:

$$
t_{w}=n \times t_{p}
$$

Where $t_{w}$ is the wait time (delay), $n$ the number of frames to wait for and $t_{p}$ the time needed by the coordinator to process one frame.

\section{B. Proposed Order}

A WVSN designed for image transmission is considered by modifying the S-MAC protocol to introduce a priority bit relating to the criticality of the monitored event. These critical data are selected by priority bit of the Beacon band. By this procedure, the source node resides in idle mode and this will decrease the power consumption. Thus, if the priority bit is set to 0 , the data sent is critical and should be processed in the first place on the coordinator. Otherwise, the data is not critical and can wait normally in the queue of the coordinator.

\begin{tabular}{|c|c|c|c|c|}
\hline Bytes $: 2$ & 1 & 4 to 20 & variable & 2 \\
\hline $\begin{array}{c}\text { Frame } \\
\text { control }\end{array}$ & $\begin{array}{c}\text { Data } \\
\text { sequence } \\
\text { number }\end{array}$ & $\begin{array}{c}\text { Address } \\
\text { information }\end{array}$ & Data payload & $\begin{array}{c}\text { Frame } \\
\text { check } \\
\text { sequence } \\
\text { MAC } \\
\text { footer }\end{array}$ \\
\hline \multicolumn{3}{|c|}{ MAC header } & MAC Payload & \\
& Priority NAV \\
& $\begin{array}{l}\text { 0: Critical Data } \\
\text { 1: Non Critical Data }\end{array}$
\end{tabular}

Fig. 3. Frame Format
Fig 3 shows the frame format for the S-MAC protocol. Adding the priority bit in the beacon frame of S-MAC protocol, helps to detect the critical data at the reception of the packet. The coordinator can easily process the critical packets before the non critical ones, which can lead to quicker reactions and energy savings due to the organization of the network.

\section{Conceptual Analysis}

In this part the conceptual analysis and the theoretical approach are explained. The different steps of the simulation are presented.

\section{A. Scenario: Implementation of the Priority-SMAC protocol}

Let's consider a WSN in star topology consisting of a coordinator as a central unit and 5 sensor-nodes (OMNeT++). Step 1: Configuration Phase - All Sensor Nodes are ready to transmit their data to the destination node. Before that, they add a tag frame with data packets and the priority bit of this tag frame is decided in the following steps.

Step 2: At the source node - Multiple nodes transmit their data by setting their priority bit to 0 or 1 . If the data is more critical, the priority bit is set to 0 , otherwise less critical data are sent and the priority bit is set to 1 . Criticality is determined based on the MASRA similarity approach.

Step 3: At the receiving node: according to the beacon frame priority bit: If higher priority data are obtained, these data are sent on the queue. The receiver notifies the transmitter having the highest priority with a beacon frame and the latter transmits the data. The receiver node, coordinator, in our work is a dual core 2 GB Ram PC. It is 4 times more powerful than a raspberry pi 3 which can be used as the sensor node where a camera can be installed. This coordinator, applies the simplest algorithm just to save the images/frames on the HDD (hard disk drive). This process, to save an image, takes $50 \mathrm{~ms}$, so $t_{p}=50 \mathrm{~ms}$.

\section{EXPERIMENTAL RESULTS}

This section consists in the presentation of our experimentations and simulations that were undertaken to validate our technique.

\section{A. Real Experimentations}

Our network is described in figure 4, every sensor-node is connected directly to a coordinator. In this work, the MACLayer protocol is the IEEE 802.11 for the communication between the sensor nodes and the coordinator. 6 raspberry pi of the $3^{\text {rd }}$ generation represent the sensor nodes, and a core 2 duo $2.0 \mathrm{GHz} 2 \mathrm{~GB}$ RAM PC serves as the coordinator. C++ language on Open $\mathrm{CV}$ has been used to configure and program the raspberry pi. The images in our experimentations are color images of $23 \mathrm{~KB}$ and $240 \times 320$ each. The MASRA algorithm was implemented on every rasbperry pi for data reduction on the sensing and transmission phases on the sensor node level to reduce the energy consumption [19]. The network operates periodically, every sensor sends the first frame of every period 
when no intrusion is detected. In this approach, a period is equal to 1 second. The maximum frame rate for the nodes is $15 \mathrm{FR} / \mathrm{s}$ (Frames per second), and the minimum frame rate that should be adopted when on idle mode (no intrusion) is 1 $\mathrm{FR} / \mathrm{s}$.

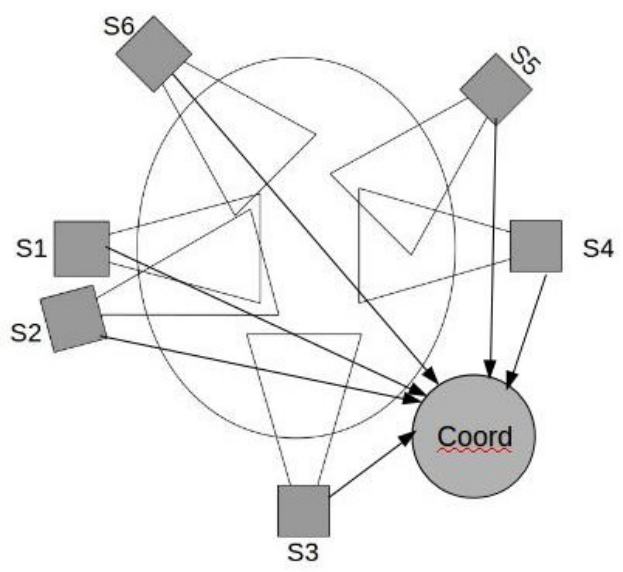

Fig. 4. Experimental Network

On the coordinator (pc), a simple algorithm to save the images on the HDD is adopted. It does no need any exceptional processing. During the idle phase, when all the nodes have a Frame Rate $F R=1$ frame per second, they send the first frame of every period, the coordinator receives 6 frames at the same time. Saving an image on the HDD is taking $t_{p}=50 \mathrm{~ms}$ (if an algorithm is implemented on the coordinator this delay increases directly). To save the $6^{\text {th }}$ frame sent from S6, we should wait $t_{w}=5 \times t_{p}=250 \mathrm{~ms}$ to save the other five frames before saving the $6^{t h}$.

This delay causes a late detection of an intrusion while on the idle mode (Frame Rate $=1 \mathrm{FR} / \mathrm{s}$ ) before increasing the frame rate of the sensors as in [19]. A late detection on the coordinator causes a late reaction from the coordinator.

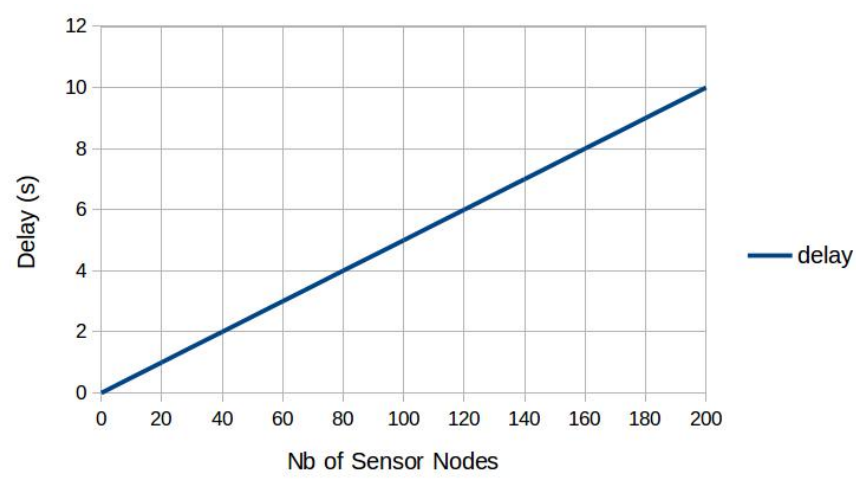

Fig. 5. The Correlation Between the Delay and the Number of Sensors
Figure 5 shows the effect of the number of sensor-nodes in an area of interest on the delay. This delay can reach a maximum of 10 seconds $\left(t_{w}=200 \times t_{p}\right)$ if the area of interest is constituted of 200 sensor-nodes and the $200^{t h}$ sensor detects the intrusion in the first place. For an average walking speed of $5 \mathrm{~km} / \mathrm{h}$, a distance of 14 meters can be covered by the walking person without any reaction from the coordinator (alerting other sensors, alerting the team in charge of the network, ...). The relation between different speeds of intrusions and non reactable distances are shown in table I.

TABLE I

Non Reactable Distance Depending on the Speed of the INTRUSION

\begin{tabular}{cccc}
\hline Event & Speed $(\mathrm{km} / \mathrm{h})$ & Delay $(\mathrm{s})$ & Distance $(\mathrm{m})$ \\
\hline Walking & 5 & 10 & 14 \\
Biking & 15 & 10 & 42 \\
Low Speed Vehicule & 40 & 10 & 111 \\
Medium Speed Vehicule & 60 & 10 & 167 \\
High Speed Vehicule & 80 & 10 & 223 \\
\hline
\end{tabular}

To avoid this delay, our approach seeks to add a priority parameter for the S-MAC protocol as mentioned in the theoretical part of this paper. This priority parameter prevents the delay on the coordinator level, the first frame with a priority 0 will be processed directly before all the other frames. If any algorithm for the reaction is there, the coordinator will react accordingly without waiting in the queue. For this purpose, Castalia and INET simulators were used for sensor networks, both simulators are based on the OMNeT++ platform.

\section{B. Network Development}

In order to implement the network both frameworks were used to access the different modules as shown in figure 6 . On the one hand the battery module in INET to calculate the energy and the creation of the sensor node as the central node. On the other hand, the priority SMAC protocol is integrated in Castalia. Our network has been initialized using Star Topology. The size of each packet in the simulations is equal to $23 \mathrm{~KB}$.

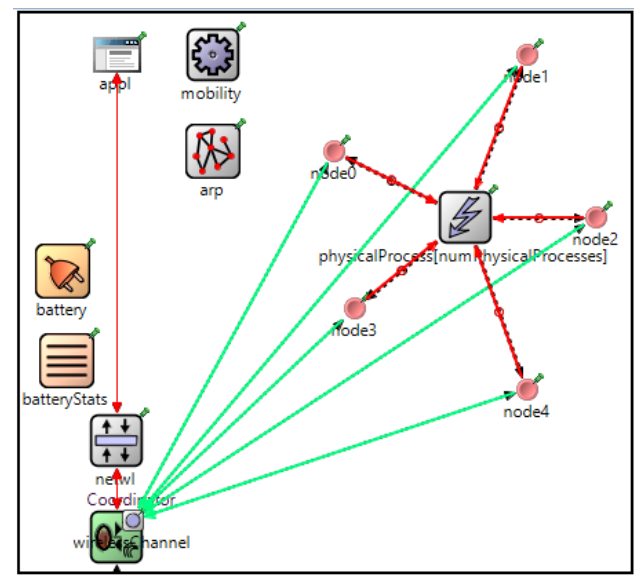

Fig. 6. Network development using Castalia and INET 
Every node is composed of different modules: ressource manager, communication, sensing, application.

\section{Delay Solution}

By studying the frames sent from the $5^{\text {th }}$ sensor (node4), the delay before being processed by the coordinator is close to $5 \mathrm{~ms}$ when the packet has a priority bit $=0$ (critical frame), which is not the case if the priority bit is not added (delay $=250$ $\mathrm{ms})$.

\section{CONCLUSION}

In this paper, the issue at stake is how to save energy by adopting MASRA algorithm from [19] while detecting the intrusion on the coordinator quicker than usual by adapting the S-MAC protocol used for sensor networks. The enhancement of the S-MAC protocol removes the delay on the queue for the critical frames. The first critical frame on the network on the idle mode must be processed in the first place on the coordinator without any delay. This delay can cause the slower detection of an intrusion on the coordinator level, it leads to a slower reaction by the coordinator. In other words, the delay weakens and affects the surveillance system negatively. Energy consumption in sensor networks is a constraint that has changed many considerations for these networks. This paper focusess on the applications of WSN using the imagery and the difficulties generated by the latter because of the large amount of data contained in the representation of an image. As a result, first it was found that the energy consumption is reduced on the sensing phase and the transmission phase [19], and the delay is totally removed from the queue of the coordinator once the first critical frame is detected. Those results validate our priority SMAC protocol for better surveillance, quicker detection and quicker reaction on the coordinator level. In our future work, we plan to work on the security in the WVSN while detecting all the intrusion on time and respecting the energy consumption.

\section{ACKNOWLEDGEMENT}

This project has been performed in cooperation with the Labex ACTION program (contract ANR-11-LABX-0001-01).

\section{REFERENCES}

[1] Tarek AlSkaify, Boris Bellalta, Manel Guerrero Zapata, and Jose M.Barcelo Ordinas. Energy efficiency of mac protocols in low data rate wireless multimedia sensor networks: a comparative study. ADHOC Networks, pages 1-17, December 2016.

[2] Ian F Akyildiz, Tommaso Melodia, and Kaushik R Chowdhury. A survey on wireless multimedia sensor networks. Computer networks, 51(4):921-960, 2007.

[3] Pei Huang, Li Xiao, Soroor Soltani, Matt w. Wutka, and Xi Ning. The evolution of mac protocols in wireless sensor networks: a survey. IEEE Communications Surveys and Tutorials, 15(1):101-120, 2013.

[4] Koen Langendoen and Andreas Meier. Analyzing mac protocols for low data-rate applications. ACM Trans. Sen. Netw., 7(1):10:1-10:34, August 2010.

[5] Cristina Cano, Boris Bellalta, Anna Sfairopoulou, and Miquel Oliver Low energy operation in wsns: A survey of preamble sampling mac protocols. Computer Networks, 55(15):3351 - 3363, 2011.
[6] Joseph Polastre, Jason Hill, and David Culler. Versatile low power media access for wireless sensor networks. In Proceedings of the 2Nd International Conference on Embedded Networked Sensor Systems, SenSys '04, pages 95-107, New York, NY, USA, 2004. ACM.

[7] Michael Buettner, Gary V. Yee, Eric Anderson, and Richard Han. Xmac: A short preamble mac protocol for duty-cycled wireless sensor networks. In Proceedings of the 4th International Conference on Embedded Networked Sensor Systems, SenSys '06, pages 307-320, New York, NY, USA, 2006. ACM.

[8] Yanjun Sun, Omer Gurewitz, and David B. Johnson. Ri-mac: A receiverinitiated asynchronous duty cycle mac protocol for dynamic traffic loads in wireless sensor networks. In Proceedings of the 6th ACM Conference on Embedded Network Sensor Systems, SenSys '08, pages 1-14, New York, NY, USA, 2008. ACM.

[9] Lei Tang, Yanjun Sun, Omer Gurewitz, and David B. Johnson. Pw-mac: An energy-efficient predictive-wakeup mac protocol for wireless sensor networks. In 2011 Proceedings IEEE INFOCOM, pages 1305-1313, April 2011.

[10] Wei Ye, John Heidemann, and Deborah Estrin. Medium access control with coordinated adaptive sleeping for wireless sensor networks. IEEE/ACM Trans. Netw., 12(3):493-506, June 2004.

[11] Tijs van Dam and Koen Langendoen. An adaptive energy-efficient mac protocol for wireless sensor networks. In Proceedings of the 1st International Conference on Embedded Networked Sensor Systems, SenSys '03, pages 171-180, New York, NY, USA, 2003. ACM.

[12] L.F.W. van Hoesel and Paul J.M. Havinga. A Lightweight Medium Access Protocol (LMAC) for Wireless Sensor Networks: Reducing Preamble Transmissions and Transceiver State Switches, pages 205208. Society of Instrument and Control Engineers (SICE), 2004.

[13] Wen-Zhan Song, Renjie Huang, Behrooz Shirazi, and Richard LaHusen Treemac: Localized tdma mac protocol for real-time high-data-rate sensor networks. Pervasive and Mobile Computing, 5(6):750 - 765, 2009. PerCom 2009.

[14] Yang Liu, I. Elhanany, and Hairong Qi. An energy-efficient qosaware media access control protocol for wireless sensor networks. In IEEE International Conference on Mobile Adhoc and Sensor Systems Conference, 2005., pages 3 pp.-191, Nov 2005.

[15] Hoon Kim and Sung-Gi Min. Priority-based qos mac protocol for wireless sensor networks. In 2009 IEEE International Symposium on Parallel Distributed Processing, pages 1-8, May 2009.

[16] Zhenzhen Liu and I. Elhanany. Rl-mac: A qos-aware reinforcement learning based mac protocol for wireless sensor networks. In 2006 IEEE International Conference on Networking, Sensing and Control, pages 768-773, 2006.

[17] Bilel Nefzi and Ye-Qiong Song. Qos for wireless sensor networks: Enabling service differentiation at the mac sub-layer using cosens. Ad Hoc Networks, 10(4):680 - 695, 2012. Advances in Ad Hoc Networks (II).

[18] M. Aykut Yigitel, Ozlem Durmaz Incel, and Cem Ersoy. Qos-aware mac protocols for wireless sensor networks: A survey. Computer Networks, 55(8): 1982 - 2004, 2011

[19] C. Salim, A. Makhoul, R. Darazi, and R. Couturier. Combining frame rate adaptation and similarity detection for video sensor nodes in wireless multimedia sensor networks. IWCMC, pages $327-332$, 2016. 